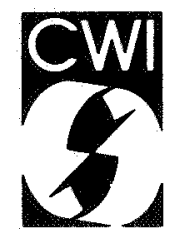

Centrum voor Wiskunde en Informatica Centre for Mathematics and Computer Science

J.W. de Bakker, J.J.Ch. Meyer, E.R. Olderog, J.I. Zucker

Transition systems, infinitary languages and the semantics of uniform concurrency 


\title{
Transition Systems, Infinitary Languages and the Semantics of Uniform Concurrency
}

\author{
J.W. de Bakker \\ Centre for Mathematics and Computer Science, Amsterdam
}

J. J.Ch. Meyer

Free University of Amsterdam

E. R. Olderog

Christian-Albrechts-Universität, Kiel

J.I. Zucker

State University of New York, Buffalo

\begin{abstract}
Transition systems as proposed by Hennessy \& Plotkin are defined for a series of three languages featuring concurrency. The first has shuffle and local nondeterminancy, the second synchronization merge and local nondeterminacy, and the third synchronization merge and global nondeterminacy. The languages are all uniform in the sense that the elementary actions are uninterpreted. Throughout, infinite behaviour is taken into account and modelled with infinitary languages in the sense of Nivat. A comparison with denotational semantics is provided. For the first two languages, a linear time model suffices; for the third language a braching time model with processes in the sense of DeBakker \& Zucker is described. In the comparison an important role is played by an intermediate semantics in the style of Hoare \& Olderog's specification oriented semantics. A variant on the notion of ready set is employed here. Precise statements are given relating the various semantics in terms of a number of abstraction operators.

1980 Mathematics Subject Classification: 68B10, 68C01.

1982 CR Categories: D.3.1, F.3.2, F.3.3.

$$
690,6973,69 F 3
$$

Key Words \& Phrases: concurrency, operational semantics, denotational semantics, transition systems, uniform languages, infinitary languages, shuffle, synchronization, local nondeterminacy, global nondeterminacy, linear time, branching time, specification-oriented semantics, ready set.

Notes:

1. The research of J.W. de Bakker is partially supported by ESPRIT project 415: Parallel Architectures and Languges;

2. This report will be published in the Proceedings $17^{\text {th }}$ ACM Symposium on Theory of Computing.
\end{abstract}


1. INTRODUCTION

our paper aims at presenting a thorough study of the semantics of a number of concepts in concurrency. We concentrate on shuffle and synchronization merge, local and global nondeterminacy, and deadlocks. Somewhat more specifically, we provide a systematic analysis of these concepts by confronting, for three sample languages, semantic techniques inspired by earlier work due to Hennessy and Plotkin $([13,20])$ proposing an operational approach, De Bakker et al. $([3,4,5,6])$ for a denotational one, and the oxford School $([8,18,19,21])$ serving - for the purposes of our paper - an intermediate role.

Our operational semantics is based on transition systems ([14]) as employed successfully in $[13,20]$; applications in the analysis of proof systems were developed by Apt $[1,2]$. Compered with previous instances, our definitions exhibit various novel features: (i) the use of a model involving languages with finite and infinite words (cf. Nivat [17]); (ii) the use of full recursion (based on the copy rule) rather than just iteration; (iii) an appealingly simple treatment of synchronization; (iv) a careful distinction between local and global nondeterminacy; (v) the restriction to uniform concurrency.

Throughout the paper we only consider uniform statements: by this we mean an approach at the schematic level, leaving the elementary actions uninterpreted and avoiding the introduction of notions such as assignments or states. Many interesting issues arise at this level, and we feel that it is advantageous to keep questions which arise after interpretation for a treatment at a second level bnot dealt with in our paper).
We shall study three languages in increasing order of complexity:

$L_{0}$ : shuffle (arbitrary interleaving) + local nondeterminacy (section 2)

$L_{1}$ : synchronization merge + local nondeterminacy (section 3)

$L_{2}$ : synchronization merge + global nondeterminacy (section 4) For $L_{i}$ with typical elements $s$, we shall present transition system $\mathrm{T}_{i}$ and define an induced operational semantics $0_{i}[s], i=0,1,2$. We shall also define three denotational semantics $D_{i}[s]$ based, for $i=0,1$ on the "linear time" (LT) model which employs sets of sequences and, for $i=2$, on the "branching time" (BT) model employing processes (commutative trees, with sets rather than multisets of successors for any node, and with certain closure properties) of $[3,4,5]$. Throughout our paper we provide $D_{i}$ only for $L_{i}$ when restricted to guarded recursion (each recursive call has to be preceded by some elementary action); we then have an attractive metric setting with unique fixed points for contractive functions based on Banach's fixed point theorem. (Our $\mathrm{O}_{i}$ do assign meaning to the unguarded case as well.)

our main question can now be posed: Do we have that

(1.1) $O_{i}\left[s \rrbracket=D_{i}[s]\right.$ We shall show that (1.1) only holds for $i=0$. For the more sophisticated languages $L_{i}, i=1,2$, we cannot prove (1.1). In fact, we can even show that there exists no $D_{i}$ satisfying $(1.1), i=1,2$. Rather than trying to modify $O_{i}$ (thus spoiling its intuitive operational character) we propose to replace $(1.1)$ by 
(1.2) $0_{i} \llbracket s \rrbracket=\alpha_{i}\left(\mathcal{D}_{i} \llbracket s \rrbracket\right)$

where $\alpha_{i}, i=1,2$, is an abstraction operator which forgets some information present in $D_{i}[s]$. The proof of $(1.2)$ requires an interesting technique of introducing a transition based intermediate semantics $I_{i}[s]$. For $i=1$ we shall show that $I_{i} \llbracket s \rrbracket=D_{i} \llbracket s \rrbracket$. Next, we introduce our first abstraction operator $\alpha_{1}$ (turning each failing communication into an indication of failure and deleting all subsequent actions) and prove that $0_{1} \prod_{s} \prod=\alpha_{1}\left(I_{1} \llbracket s \rrbracket\right)$.

The case $i=2$ is more involved, because $L_{1}$ has local, and $L_{2}$ global nondeterminacy. Consider a choice a or $c$, where $a$ is some autonomous action and $c$ needs a parallel $\bar{c}$ to communicate. In the case of local nondeterminacy (written as a $u c$ ) both actions may be chosen; in the global nondeterminacy case (written as $a+c,+$ as in CCS [16]) $c$ is chosen only when in some parallel compound $\bar{c}$ is ready to execute. Therefore, $L_{1}$ and $L_{2}$ exhibit different deadlock behaviours. $\mathrm{O}_{2}$ is based on the transition system $\mathrm{T}_{2}$ which is a refinement of $T_{1}$, embodying a more subtle set of rules to deal with nondeterminacy. The denotational semantics $D_{2}$ is as in $[3,4,5]$. In order to relate $D_{2}$ and $O_{2}$ we introduce the notion of readies and associated intermediate semantics $I_{2}$, inspired by ideas as described in $[8,18,19,21]$. $I_{2}$ involves an extension of the LT model with some branching information (though less than the full BT model) which is amenable to a treatment in terms of transitions. The proof of the desired result is then obtained by relating the semantics $\mathrm{O}_{2}, \mathrm{O}_{2}$ and $\mathrm{I}_{2}$ by a careful choice of suitable abstraction operators.

As main contributions of our paper we see s.
1. The three transition systems $\mathrm{T}_{i}$, in particular the refinement of $\mathrm{T}_{1}$ into $\mathrm{T}_{2}$.

2. The systematic treatment of the denotational semantics definitions (for the guarded case) together with the settiing of the relationship $0_{i}=\alpha_{i} \circ D_{i} \cdot\left(\alpha_{0}\right.$ identity $)$

3. Clarification of local versus global nondeterminacy and associated deadlock behaviour.

4. The intermediate semantics $I_{1}$ and, in particular, $I_{2}$.

2. THE LANGUAGE $L_{0}$ : SHUFFLE AND LOCAL NONDETERMINACY Let $A$ be a finite alphabet of elementary actions with $a \in A$. Let $x, y$ be elements of the alphabet stmv of statement variables (used in fixed point constructs for recursion). As syntax for $s \in L_{0}$ we give

$$
s:=a\left|s_{1} ; s_{2}\right| s_{1} \cup s_{2}\left|s_{1} \| s_{2}\right| x \mid u x[s] .
$$

A term $\mu x[s]$ is a recursive statement. For example, according to the definitions to be proposed presentiy, the intended meaning of $\mu \times[(a ; x) \cup b]$ is the set $\left\{a^{\omega}\right\} \cup a^{*} \cdot b$, with a the infinite sequence of $a^{\prime} s$.

2.1. The transition system $T_{0}$ Let $A^{\text {tr }}=$ df. $A^{*} \cup A^{\omega} \cup A^{*} \cdot\{1\}$, with $A^{*}$ the set of all finite words over $A, A^{*}$. $\{1\}$ the set of all (finite) unfinished words over $A$, and $A^{\omega}$ the set of all infinite words over $A$, and $\perp \notin A$. Let $w, u, v$ denote elements of $A^{\text {tr }}$, and let $\lambda$ be the empty word. We define $1 . \mathrm{w}=\perp$ for all $\mathrm{w}$.

A configuration is a pair $\langle s, w\rangle$ or just a word $w$. A transition relation is a binary relation over configurations. A transition is a formula $\left\langle s, w^{\prime}\right\rangle \rightarrow\left\langle s^{\prime}, w^{\prime}\right\rangle$ or $\left\langle s, w^{\prime}\right\rangle+w^{\prime}$ denoting an element of a transition relation. A transition system is a formal deductive system for proving transitions 
based on axioms and rules. Using a self-explanatory notation, axioms have the format $1 \rightarrow 2$, rules have the format $\frac{1 \rightarrow 2}{3 \rightarrow 4}$. Also, $1 \rightarrow 2 \mid 3$ abbreviates $1 \rightarrow 2$ and $1 \rightarrow 3$, and $\frac{1+2}{4+5} \mid \frac{3}{6}$ abbreviates $\frac{1 \rightarrow 2}{4 \rightarrow 5}$ and $\frac{1 \rightarrow 3}{4 \rightarrow 6}$. For a transition system $T, T \vdash(1 \rightarrow 2)$ expresses that transition $1 \rightarrow 2$ is deducible from system $T$.

We now present the transition system $\mathrm{T}_{0}$ for $L_{0}:$

$\left\langle s, w>\rightarrow w, w \in A \cup A^{*} .\{\perp\}\right.$. For $w \in A^{*}$ we put

(elementary action)

$\langle a, w\rangle \rightarrow \mathrm{w} \cdot \mathrm{a}$

(local nondeterminacy)

$$
\left\langle s_{1} \cup s_{2}, w\right\rangle \rightarrow\left\langle s_{1}, w\right\rangle \mid\left\langle s_{2}, w\right\rangle
$$

(recursion)

$$
\langle\mu x[s], w\rangle \rightarrow\langle s[\mu x[s] / x], w\rangle
$$

where, in general, $s[t / x]$ denotes substitution

of $t$ for $x$ in $s$

(sequential composition)

$$
\frac{\left\langle s_{1}, w_{1}\right\rangle \rightarrow w^{\prime} \mid\left\langle s^{\prime}, w^{\prime}\right\rangle}{\left\langle s_{1} ; s_{2}, w_{1}\right\rangle \rightarrow\left\langle s_{2}, w^{\prime}\right\rangle \mid\left\langle s^{\prime} ; s_{2}, w^{\prime}\right\rangle}
$$

(shuffle)

$$
\begin{aligned}
& \left\langle s_{1}, w_{1}\right\rangle \rightarrow w^{\prime} \mid\left\langle s^{\prime}, w^{\prime}\right\rangle \\
& \left\langle s_{1} \| s_{2}, w_{1}\right\rangle \rightarrow\left\langle s_{2}, w^{\prime}\right\rangle \mid\left\langle s^{\prime} \| s_{2}, w^{\prime}\right\rangle \\
& \left\langle s_{1}, w_{1}\right\rangle \rightarrow w^{\prime} \mid\left\langle s^{\prime}, w^{\prime}\right\rangle \\
& \left\langle s_{2} \| s_{1}, w_{1}\right\rangle \rightarrow\left\langle s_{2}, w^{\prime}\right\rangle \mid\left\langle s_{2} \| s^{\prime}, w^{\prime}\right\rangle
\end{aligned}
$$

2.2. The operational semantics $0_{0}$

We show how to obtain $O_{0}$ from $T_{0}$. We define

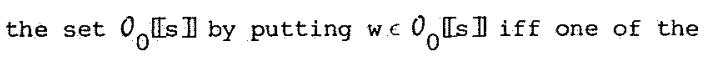
following three conditions is satisfied (always taking $\left.\left\langle\mathrm{s}_{0}, \mathrm{w}_{0}\right\rangle=\mathrm{df} \cdot\langle\mathrm{s}, \lambda\rangle\right)$ :

1. There is a finite sequence of $T_{0}$-transitions

$$
<s_{0}, w_{0}>\rightarrow \ldots \rightarrow<s_{n}, w_{n}>\rightarrow w
$$

2. There is an infinite sequence of $\mathrm{T}_{0}$-transitions $\left\langle s_{0}, w_{0}\right\rangle+\ldots \rightarrow\left\langle s_{n}, w_{n}\right\rangle \rightarrow\left\langle s_{n+1}, w_{n+1}\right\rangle \rightarrow \ldots$ \left. where the sequence ${\left\langle w_{n}\right.}_{n}\right\rangle_{n=0}^{\infty}$ is infinitely often increasing, and $w=\sup _{n} w_{n}$ (sup with respect to prefix ordering).

3. There is an infinite sequence as in 2 , but now

$w_{n+k}=w_{n}$ for some $n$ and $a l l k \geq 0$ and $w=w_{n} \cdot 1$ Examples. $\mathrm{O}_{0} \mathbb{L}\left(\mathrm{a}_{1} ; \mathrm{a}_{2}\right) \| \mathrm{a}_{3} \rrbracket=\left\{\mathrm{a}_{1} \mathrm{a}_{2} \mathrm{a}_{3}, \mathrm{a}_{1} \mathrm{a}_{3} \mathrm{a}_{2}, \mathrm{a}_{3} \mathrm{a}_{1} \mathrm{a}_{2}\right\}$ $0_{0} \llbracket \mu x[(a ; x) \cup b] \rrbracket=a^{*} \cdot b \cup\left\{a^{\omega}\right\}, 0_{0} \llbracket u x[(x ; a) \cup b] \rrbracket=$ $=b \cdot a^{*} u\{1\}$.

Remark: Observe that systems such as $\mathrm{T}_{0}$ are used to deduce (one step) transitions $1 \rightarrow 2$. Sequences of such transitions are used only to define $0_{0} \llbracket . \rrbracket$. 2.3. The denotational semantics $D_{0}$ We introduce a denotational semantics $D_{0}$ for the language $L_{0}$ based on an approach using metric spaces (rather than the more customary cpo's) as underlying structure. This section is based on [3]; for the topology see [10]. We recall that $D_{i}$ is defined only for the guarded case: Each $\mu x[s]$ is such that all free occurrences of $x$ in $s$ are sequentially preceded by some statement.

For $u \in A^{t r}$ let $u[n], n \geq 0$, be the prefix of $u$ of length $n$ if this exists, otherwise $u[n]=u$. E.g., $a b c[2]=a b, a b c[5]=a b c$. We define $a$ natural metric $d$ on $A^{\text {tr }}$ by putting

$$
d(u, v)=2^{-\max \{n \mid u[n]:=v[n]\}}
$$

with the understanding that $2^{-\infty}=0$. For example, $d(a b c, a b d)=2^{-2}, d\left(a^{n}, a^{\omega}\right)=2^{-n}$. We have that $\left(A^{t r}, d\right)$ is a complete metric space. For $x \subseteq A^{\text {tr }}$ we put $x[n]=\{u[n] \mid u \in X\}$. A distance $\hat{d}$ on subsets $X, Y$ of $A^{t r}$ is defined by

$$
\left.\hat{d}(X, Y)=2^{-\max \{n \mid X[n]}=Y[n]\right\}
$$

Let $\mathrm{C}$ denote the collection of all closed subsets of $A^{\text {tr }}$. It can be shown that $(C, \hat{d})$ is a complete metric space. A sequence $\left\langle x_{i}\right\rangle_{i=0}^{\infty}$ of elements of $\mathcal{C}$ is a Cauchy sequence whenever $\forall \varepsilon>0 \quad \exists N \forall n, m \geq N\left[\hat{d}\left(x_{n}, x_{m}\right)<\varepsilon\right]$. For $\left\langle x_{i}\right\rangle_{i}$ a Cauchy sequence, we write $\lim _{i} x_{i}$ for its limit (which belongs to $\mathcal{C}$ by the completeness property) 
A function $\phi:(C, \hat{d}) \rightarrow(C, \hat{d})$ is called contracting whenever, for all $\mathrm{X}, \mathrm{Y}, \hat{\mathrm{d}}(\phi(\mathrm{X}), \phi(\mathrm{Y})) \leq \alpha$. $\hat{d}(X, Y)$, for some real number $\alpha$ with $0 \leq \alpha<1$. A classical theorem due to Banach states that in any complete metric space, a contracting function has a unique fixed point obtained as $\lim _{i} \phi^{i}\left(x_{0}\right)$ for arbitrary starting point $\mathrm{x}_{0}$.

we now define the operations ., $u\|\|$ on $C$ in the following way:

a. $X, Y \subseteq A^{*} \cup A^{*} .\{\perp\}$. For $X . Y$ and $X \cup Y$ we adopt the usual definitions (including the clause 1. $u=\perp$ for all $u$ ). For $x \| y$ we introduce as auxiliary operator the so-called left-merge $\mathbb{L}$ (from [7]). We put $X \| Y=(X \Perp Y) \cup(Y \amalg X)$, where $\mathbb{L}$ is given by $X \mathbb{L} Y=U\{u \amalg Y \mid u \in X\}, E \amalg L=Y$, $a \Perp Y=a . Y, L L Y=\{\perp\}$, and $(a . u)\lfloor Y=$ a. $(\{u\} \| y)$.

b. $X, Y \in \mathcal{C}, X . Y$ do not consist of finite words only. Then $\mathrm{X}$ op $\mathrm{Y}=\lim _{\mathrm{i}}(\mathrm{X}[\mathrm{i}]$ op $\mathrm{Y}[\mathrm{i}])$, for $o p \in\{,, u, \|\}$. In [3] we have shown that this definition is well-formed and preserves closed sets, and the operations are continuous (for this finiteness of $A$ is necessary).

we proceed with the definition of $D_{0} \llbracket s \rrbracket$ for $s \in L_{0}$. We introduce the usual notion of environment which is used to store and retrieve meanings of statement variables. Let $\Gamma=S t m v \rightarrow C$ be the set of environments, and let $\gamma \in \Gamma$. We write $\gamma^{\prime}=d f . \gamma\langle x / x\rangle$ for a variant of $\gamma$ which is like $\gamma$ but such that $\gamma^{\prime}(x)=x$. We define $D_{0}: L_{0} \rightarrow(\Gamma+C)$ as followe:

DEFINTTION.

$D_{0} \llbracket a \rrbracket(\gamma)=\{a\}, D_{0} \llbracket s_{1} \circ p s_{2} \rrbracket(\gamma)=D_{0} \llbracket s_{1} \rrbracket(\gamma) o p$ $D_{0} \llbracket s_{2} \rrbracket(\gamma)$, for $o p \in\{,, U, \|\}, D_{0} \llbracket x \rrbracket(\gamma)=\gamma(x)$, and

$$
\begin{aligned}
& D_{0} \llbracket u x[s] \rrbracket(\gamma)=1 \mathrm{im}_{i} x_{i}, \text { where } x_{0}=\{\perp\} \text { and } \\
& x_{i+1}=D_{0} \llbracket s \rrbracket\left(\gamma<x_{i} / x>\right)
\end{aligned}
$$

By the guardedness requirement, each function $\phi=\lambda x . D_{0}[s \rrbracket(\gamma<x / x\rangle)$ is contracting, $\left\langle x_{i}\right\rangle_{i}$ is a Cauchy sequence, and $\lim _{i} x_{i}$ equals the unique fixed point of $\phi$.

Remark. An order-theoretic approach to the denotational model is also possible (cf. $[9,15]$ ). However, for our present purposes this has no special advantages. In fact, the order-theoretic approach does not provide a direct treatment for the unguarded case either, it seems to require a contractivity argument for uniqueness of fixed points just as well, and, last but not least, as far as we know, it cannot be used as a basis for the $B T$ model.

2.4. Relationship between $O_{0}$ and $O_{0}$.

We shall prove (for statements $s$ without free statement variables, and omitting $\gamma$ ).

THEOREM 2.1. $O_{0}=D_{0}$.

The proof relies on four lemmas.

LEMMA 2.2, $0_{0}$ is homomorphic over ..v, $\|$.

LEMMA 2.3. (guarded case only). Consider a $\mu$-term $\mu x[s]$. Let $\Omega$ be the (auxiliary) statement such that $\langle\Omega, w\rangle \rightarrow$ w.L. Let $s^{(0)}=\Omega, s^{(n+1)}=s\left[s^{(n)} / x\right]$. Then $0_{0}\left[\mu x[s] \rrbracket=\lim _{n} 0_{0}\left[s s^{(n)} \rrbracket\right.\right.$.

PROOF. This involves a detailed analysis of transition sequences; it introduces in particular the notion of truncating a sequence after $n$ applications of the recursion axiom involving the considered $\mu$-term.

LEMMA 2.4. (guarded case only). For each $s, 0_{0} \llbracket s \mathbb{D}$ is a closed set.

Caution. This is not true for the unguarded case. For example, $\mathcal{O}_{0} \llbracket \mu x[(x ; a) \cup b] \rrbracket=\{1\} \cup b . a^{*}$. This set is not closed since its limit point ba ${ }^{\omega}$ is not in it.

LEMMA 2.5. (this is the crucial lemma relating $O_{0}$ 
and $\left.D_{0}\right)$. Let $\operatorname{var}(s) \subseteq\left\{x_{1}, \ldots, x_{n}\right\}$. Let $t_{i}$ be without free statement variables, and let $x_{i}=0_{0} \llbracket t_{i} \rrbracket, i=1, \ldots, n$. Then

$$
D_{0} \llbracket s \rrbracket\left(\gamma<x_{i} / x_{i}>{ }_{i=1}^{n}\right)=0_{0} \llbracket s<t_{i} / x_{i}>_{i=1}^{n} \rrbracket \text {. }
$$

PROOF. Structural induction on $s$.

3. THE LANGUAGE $L_{1}$ : SYNCHRONIZATION MERGE AND

\section{LOCAL NONDETERMINACY}

Let $A$ be a finite alphabet, let $C \subseteq A$ with $c \in C$ (the communications) and let $a \in A \backslash C$. Let there be given a bijection ${ }^{-}: \mathrm{C}+\mathrm{C}$ (matching communications à la $\operatorname{CSS} / \mathrm{CSP}$ ) with $\overline{\bar{c}}=c$. Let $\tau \in \mathrm{A}$ be a special symbol serving as a meaning for the skip statement, and let $\delta$ be an element not in $A$ indicating failure. We always have $\delta . w=\delta$. Let

$$
A_{\delta}^{\operatorname{tr}}=A^{*} \cup A^{\omega} \cup A^{*} \cdot\{\delta, 1\}
$$

$u, v, w$ now range over $A_{\delta}^{t r}$. As syntax for $s \in L_{1}$ we give

$$
s:=a|c| \underline{\text { skip }}|\underline{\text { fail }}| s_{1} ; s_{2}\left|s_{1} \cup s_{2}\right| s_{1} \| s_{2}|x| \mu x[s] .
$$

3.1. The transition system $\mathrm{T}_{1}$.

The system $T_{1}$ consists of $T_{0}$ extended with: $\langle s, w\rangle \rightarrow w$ for $w \in A^{w} \cup A^{*},\{\delta, \perp\}$. For $w \in A^{*}$ we have (communication)

$\langle c, w\rangle \rightarrow\langle\underline{f a i l}, w\rangle$ an individual communication

$$
\text { fails }
$$

(skip)

\section{$\langle$ skip, $w>+w . \tau$}

(failure)

$$
\langle\text { fail, } w\rangle \rightarrow w . \delta
$$

(synchronization)

$$
\begin{array}{ll}
\langle\mathrm{c} \| \bar{c}, \mathrm{w}\rangle & \rightarrow\langle\underline{\text { skip } ; w\rangle} \\
\left\langle\mathrm{c} ; \mathrm{s}_{1} \| \overline{\mathrm{c}}, \mathrm{w}\right\rangle & \rightarrow\left\langle\text { skip; } \mathrm{s}_{1}, \mathrm{w}\right\rangle \\
\left\langle\mathrm{c} \| \overline{\mathrm{c}} ; \mathrm{s}_{2}, \mathrm{w}\right\rangle & \rightarrow\left\langle\text { skip; } \mathrm{s}_{2}, \mathrm{w}\right\rangle \\
\left\langle\mathrm{c} ; \mathrm{s}_{1} \| \overline{\mathrm{c}} ; \mathrm{s}_{2}, \mathrm{w}\right\rangle \rightarrow\left\langle\text { skip }\left(\mathrm{s}_{1} \| \mathrm{s}_{2}\right), \mathrm{w}\right\rangle
\end{array}
$$

(commutativity and associativity of merge)

$$
\begin{aligned}
& \frac{\left\langle s_{1} \| s_{2}, w\right\rangle+\left\langle s^{\prime}, w^{\prime}\right\rangle}{\left\langle s_{2} \| s_{1}, w\right\rangle+\left\langle s^{\prime}, w^{\prime}\right\rangle} \\
& \frac{\left\langle s_{1} \|\left(s_{2} \| s_{3}\right), w\right\rangle \rightarrow\left\langle s^{\prime}, w^{\prime}\right\rangle}{\left\langle\left(s_{1} \| s_{2}\right) \| s_{3}, w^{\prime}\right\rangle\left\langle s^{\prime}, w^{\prime}\right\rangle} \text {, and symmetric. }
\end{aligned}
$$

Remark. Note that associativity/commutativity of merge are provable in $\mathrm{T}_{0}$.

3.2. The operational semantics $O_{1}$

$0_{1}[s]$ is defined similarly to $0_{0} \llbracket s \rrbracket$. Now failing communications result in $\delta$, successful communications (through the synchronization rule) in addition in $\tau$.

Examples. $O_{1}[c]=\{\delta\}, O_{1}[(a ; b) \cup(a ; c) \rrbracket=\{a b, a \delta\}$, $O_{1}[c \| \bar{c} \mathbb{D}=\{\delta, \tau\}$. We observe too many $\delta$ 's here: to do away with such appearances of deadlocks in case an alternative is present, we postulate - for the remainder of section 3 only - the axiom

(3.1) $\{\delta\} \cup x=x$, for $x \neq \emptyset$

(Formally, we should now take congruence classes in $A^{\text {tr }}$ with respect to (3.1); we do not bother to be that precise.) Taking (3.1) into account, the above examples now become $O_{1} \llbracket c \rrbracket=\{\delta\}$,

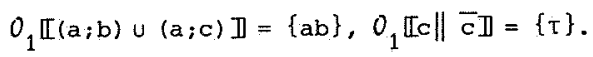
It is important to observe that the two statements $(a ; b) \cup(a ; c)$ and $a ;(b \cup c)$ obtain the same meaning by $o_{1}$. Section 4 will provide a more refined treatment.

3.3. The denotational semantics $D_{1}$. This is as in section 2,3. but extended/modified in the following way fomitting $\gamma$-arguments for simplicity):

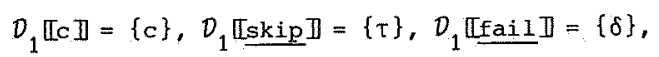
$D_{1} \llbracket s_{1}\left\|s_{2} \rrbracket=D_{1} \llbracket s_{1} \rrbracket\right\| D_{1} \llbracket s_{2} \rrbracket$, where, for $X, Y \subseteq A^{t r}$, we define $X \| Y=(X \mathbb{L} Y) \cup(Y \mathbb{L} X) \cup(X \mid Y)$. Here the

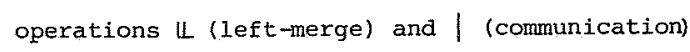
are defined as follows: First we take the case that $X, Y$ consist of finite words onl $Y$. 
$X \nVdash Y=U\{W \amalg Y \mid W \in X\}, \perp \amalg Y=\{\perp\}, \delta \Perp Y=\{\delta\}$,

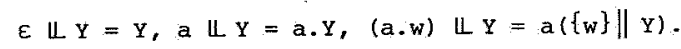
Also, $x \mid y=\{(w \mid u): w \in X, u \in Y\}$, where $\left(c \cdot w_{1}\right) \mid\left(\bar{c} \cdot u_{1}\right)=\tau \cdot\left(w_{1} \| u_{1}\right)$. Moreover, $w^{\prime} \mid u^{\prime}=\delta$ for $w^{\prime}, u^{\prime}$ not of such $a$ form. If $x$ or $x$ contains infinite words, the definition is completed by taking limits. (The definition of $x \| x$ is from [7].)

3.4. Relationship between $O_{1}$ and $D_{1}$. we do not simply have that

(*) $O_{1} \llbracket s \rrbracket=D_{1} \llbracket s \rrbracket$. (Take $\mathrm{s}=\mathrm{c}$ for a counter example. Then $O_{1} \llbracket \mathrm{c} \rrbracket=\{\delta\}$, $D_{1}[c]=\{c\}$ ). We even have that: THEOREM 3.1. There does not exist any denotational (implying compositional) semantics $D$ satisfying ( $*$ ). .The proof is based on

LEMMA 3.2. $0_{1}$ does not behave compositionally over $\|$. proof. We show that there exists no "mathematical" operator $\|_{D}$ such that $0_{1} \llbracket s_{1}\left\|s_{2} \rrbracket=0_{1} \llbracket s_{1} \rrbracket\right\|_{D}$ $\mathrm{O}_{1} \llbracket s_{2} \rrbracket$. Consider the programs $s_{1}=\mathrm{c}, s_{2}=\bar{c}$ in $L_{1}$. Then $0_{1}\left[s_{1} \rrbracket=0_{1}\left[s_{2} \rrbracket=\delta\right.\right.$. Suppose now that $\|_{D}$ exists. Then $\{\delta\}=O \Pi s_{1}\left\|s_{1} \rrbracket=O \llbracket s_{1} \rrbracket\right\|_{D} \quad O \llbracket s_{1} \rrbracket=$ $O \llbracket s_{1} \rrbracket\left\|_{D} O \llbracket s_{2} \rrbracket=O \llbracket s_{1}\right\| s_{2} \rrbracket=\{\tau\}$.

Contradiction.

We remedy this not by redefining $T_{1}$ (which adequately captures the operational intuition for $\left.L_{1}\right)$, but rather by introducing an abstraction mapping $\alpha_{1}$ such that

$$
\text { (**) } 0_{1}=\alpha_{1} \circ D_{1} \text {. }
$$

We take $\alpha_{1}=s y n_{1}$ defined by $\left(\omega \subseteq A_{\delta}^{t r}\right.$ )

$$
\begin{array}{r}
\operatorname{syn}_{I}(w)=\{w \mid w \in w \text { does not contain } c \in c\} u \\
\left\{w . \delta \mid \exists w^{\prime}, c^{\prime} \text { such that } w^{\prime} c^{\prime} \cdot w^{\prime} \in w,\right. \\
w \text { contains no } c\}
\end{array}
$$

The right-hand side of this definition should be taken with respect to $(\delta . \mathrm{w}=\delta$ and $)\{\delta\} \cup \mathrm{x}=\mathrm{X}$, $\mathrm{x} \neq \emptyset$. Informally, syn ${ }_{1}$ replaces unsuccessful synchronization by deadlock and keeps this in case there is no alternative.

We cannot prove $(* *)$ ' by a direct structural induction on $s$ (because $s n_{1}$ does not behave homomorphically). Rather, we introduce an intermediate semantics $I_{1}$ : we modify $T_{1}$ into $T_{1}^{*}$ which is the same as $T_{1}$ but for the communication axiom which now has the form (communication ${ }^{\star}$ )

$$
\langle\mathrm{c}, \mathrm{w}\rangle \rightarrow \mathrm{w} \cdot \mathrm{c}
$$

We base $I_{1}$ on $T_{1}^{*}$ just as we based $0_{1}$ on $T_{1}$. We can now prove

LEMMA 3.3. For all $s, s^{\prime} \in L_{1}$ and $w, w^{\prime} \in(A \backslash C)^{*}$

$$
\begin{aligned}
& T_{1} \vdash\left\langle s, w^{\prime}\right\rangle+w^{\prime} \mid\left\langle s^{\prime}, w^{\prime}\right\rangle \\
& \text { iff } \\
& T_{1}^{*}+\left\langle s, w^{*}+w^{\prime}\right|\left\langle s^{\prime}, w^{\prime}\right\rangle
\end{aligned}
$$

Proof. Structural induction on the deductions in $\mathrm{T}_{1}$ and $\mathrm{T}_{1}^{*}$.

This lemma immediately leads to THEOREM 3.4. $O_{1}[s]=\operatorname{syn}_{1}\left(I_{i}[s]\right)$

Next we show

THEOREM 3.5. $I_{1}\left[s \rrbracket=D_{1} \llbracket s \rrbracket\right.$

Proof. Combine ideas of section 2.4 with a proof that $I_{1}$ behaves compositionally over $\|$ las defined in section 3.3$)$.

Remark. This proof recalls Apt's merging lemma $[1,2]$.

By combining theorems $3.4,3.5$ we finally obtain our desired result THEOREM 3.6. $\left.O_{1}[s]=\operatorname{syn}_{1}\left(D_{1} \llbracket s\right]\right)$.

4. THE LANGUAGE $L_{2}$ : SYNCHRONIZATION MERGE AND GLOBAL NONDETERMTNACY

The syntax for $s \in L_{2}$ is given by 
$s::=a|c| \underline{\text { skip }}|\underline{\text { fail }}| s_{1} ; s_{2}\left|s_{1}+s_{2}\right| s_{1} \| s_{2}|x| \mu \times[s]$ Here "+" denotes global nondeterminacy; the notation is from CCs[16].

4.1. The transition system $\mathrm{T}_{2}$. $\mathrm{T}_{2}$ is like $\mathrm{T}_{1}$, but without the axion for local nondeterminacy, and without the axiom for communication $(\langle c, w\rangle \rightarrow\langle\underline{\text { fail }}, w\rangle)$. Additionally, we have

(global nondeterminacy)

[u-unfolding]

$\frac{\left\langle s_{1}, w\right\rangle+\left\langle s^{\prime}, w\right\rangle}{\left\langle s_{1}+s_{2}, w\right\rangle \rightarrow\left\langle s^{\prime}+s_{2}, w\right\rangle}$

[selection by elementary action]

$\frac{\left\langle s_{1}, w\right\rangle \rightarrow w^{\prime} \mid\left\langle s^{\prime}, w^{\prime}\right\rangle}{\left\langle s_{1}+s_{2}, w^{\prime}+w^{\prime}\right|\left\langle s^{\prime}, w^{\prime}\right\rangle}$, where $w^{\prime} \neq w$

[selection by communication/synchronization]

$\frac{\left\langle s_{1} \| s_{3}, w\right\rangle \rightarrow\left\langle s^{\prime}, w^{\prime}\right\rangle}{\left\langle\left(s_{1}+s_{2}\right) \| s_{3}, w^{\prime}\right\rangle \rightarrow\left\langle s^{\prime}, w^{\prime}\right\rangle}$, where the

transition in the premise involves

synchronization between actions from $s_{1}$

and $s_{3}$

[commutativity of + ]

$\frac{\left\langle s_{1}+s_{2}, w^{\prime}\right\rangle \rightarrow w^{\prime} \mid\left\langle s^{\prime}, w^{\prime}\right\rangle}{\left\langle s_{2}+s_{1}, w^{\prime}\right\rangle+w^{\prime} \mid\left\langle s^{\prime}, w^{\prime}\right\rangle}$

$\frac{\left\langle\left(s_{1}+s_{2}\right) \| s_{3}, w^{\prime}\right\rangle+w^{\prime} \mid\left\langle s^{\prime}, w^{\prime}\right\rangle}{\left\langle\left(s_{2}+s_{1}\right)|| s_{3}, w^{\prime}\right\rangle+w^{\prime} \mid\left\langle s^{\prime}, w^{\prime}\right\rangle}$

Remark. Associativity of + is derivable.

We see that global nondeterminacy is more restrictive than local nondeterminacy. In fact,

$\left\langle s_{1}+s_{2}, w^{\prime}\right\rangle+w^{\prime} \mid\left\langle s^{\prime}, w^{\prime}\right\rangle$ implies

$\left\langle s_{1} u s_{2}, w^{\prime}\right\rangle+w^{\prime} \dagger\left\langle s^{\prime}, w^{\prime}\right\rangle$ but not vice versa.

Example. 〈auc, $w\rangle \rightarrow^{*}$ w. $\delta,\langle a u c, w\rangle \rightarrow^{*}$ w.a, but

$\langle\mathrm{a}+\mathrm{c}, \mathrm{w}\rangle \rightarrow^{*}$ w.a only. In the case of global

nondeterminacy, the communication transitions of $\mathrm{s}_{1}+\mathrm{s}_{2}$ depend on the communication transitions of $s_{1}$ and $s_{2}$ in some global context $s_{1} \| s_{3}$ or $s_{2} \| s_{3}$.
This formalizes the communication as present in languages like CSP, ADA or OCCAM.

4.2. The operational semantics $\mathrm{O}_{2}$

$\mathrm{O}_{2}$ is derived form $\mathrm{T}_{2}$ in the usual way. In addition, however, we now have to consider the case that we have a finite sequence

$\langle s, \lambda\rangle=\left\langle s_{0}, w_{0}\right\rangle+\ldots \rightarrow\left\langle s_{n}, w_{n}\right\rangle$, with no transition $\left\langle s_{n}, w_{n}+\ldots\right.$ deducible. we then deliver $w_{n} \cdot \delta$ as

element of $\mathrm{O}_{2} \llbracket s \rrbracket$. The pair $\left\langle s_{n}, w_{n}\right\rangle$ is then called a deadlocking configuration.

Example. $\mathrm{O}_{2}[(a ; b)+(a ; c) \rrbracket=\{a b, a \delta\}$,

$\mathrm{O}_{2}[\mathrm{Ia} ;(\mathrm{b}+\mathrm{c}) \mathbb{Z}=\{\mathrm{ab}\}$.

4.3. The denotational semantics $D_{2}$.

we follow $[3,4,5]$ in introducing a branching time semantics for $L_{2}$. Let $A_{\perp}=$ df. $A \cup\{\perp\}$. Let $P_{n}$, $\mathrm{n} \geq 0$, be defined by

$$
P_{0}=P\left(A_{\perp}\right), P_{n+1}=P\left(A_{\perp} \cup\left(A_{\perp} \times P_{n}\right)\right)
$$

where $P($.$) denotes all subsets of (.), and let$ $P_{\omega}=U_{n} P_{n}$. We define a metric $d$ on $P_{\omega}$ (for its definition see $[3,4,5]$ ) and take $P$ as the completion of $\mathrm{P}_{w}$ with respect to $d$. It can be shown that $P$ satisfies the domain equation

$$
P=P_{\text {closed }}\left(A_{\perp} \cup\left(A_{\perp} \times P\right)\right)
$$

Finite elements of $P$ are, e.g., $\left\{\left[a,\left\{b_{1}\right\}\right],\left[a,\left\{b_{2}\right\}\right]\right\}$ or $\left\{\left[a,\left\{b_{1}, b_{2}\right\}\right]\right\}$. Thus, the branching structure is preserved. An infinite element is, e.g., the process $p$ which satisfies the equation $\mathrm{p}=\{[\mathrm{a}, \mathrm{p}],[\mathrm{b}, \mathrm{p}]\}$. The empty set is a process and takes the role of $\delta$. Note that in the LT framework, $\emptyset$ cannot replace $\delta$ since by the definition of concatenation (for IT) we have a. $\emptyset=\emptyset$ which is undesirable for an element modelling failure. (An action which fails should not cancel all previous actions.) In the BT framework, $\{[a, \emptyset]\}$ is a process which is indeed different from $\emptyset$. Since, clearly, $\emptyset \cup \mathrm{p}=\mathrm{p}$ for all sets (processes) $\mathrm{p}$, we can do 
without explicitly imposing a counterpart of rule (3.1) for $\delta$.

Operations ., $u, \|$, limits and continuity, fixed points of contracting operations are as in $[3,4,5]$. For example, for $p, q \in P_{\omega^{\prime}}$ we put $p \| q=(p \perp q) v(q \| p) \quad v(p \mid q)$ where $p\llcorner q=\{x \perp q: x \in p\}, a \perp q=[a, q], \perp L q=\perp$, $\left[a, p^{\prime}\right] \cup q=\left[a, p^{\prime} \| q\right]$, and $p \mid q=U(x \mid y): x \in p$, $\mathrm{y} \in \mathrm{q}\}$, where $\left[c, \mathrm{p}^{\prime}\right] \mid\left[\bar{c}, \mathrm{q}^{\prime}\right]=\left\{\left[\tau, \mathrm{p}^{\prime} \| \mathrm{q}^{\prime}\right]\right\}$, $c\left|\left[\bar{c}, q^{\prime}\right]=\left\{\left[\tau, q^{\prime}\right]\right\},\left[c, p^{\prime}\right]\right| \bar{c}=\left\{\left[\tau, p^{\prime}\right]\right\}$, $c \mid \bar{c}=\{\tau\}$, and $(x \mid y)=\emptyset$ when $x, y$ are not of one of these four forms.

It is now straightforward to define $D_{2}: L_{2} \rightarrow\left(\Gamma_{2} \rightarrow P\right)$, where $\Gamma_{2}=s t m v \rightarrow P$, by following the clauses in the definition of $D_{0}, D_{1}$. Thus we put $D_{2} \llbracket a \rrbracket(\gamma)=\{a\}, D_{2} \mathbb{I} s_{1}$ op $s_{2} \rrbracket(\gamma)=$ $D_{2} \llbracket s_{1} \rrbracket(\gamma) \circ p D_{2} \llbracket s_{2} \rrbracket(\gamma), D_{2} \llbracket x \rrbracket(\gamma)=\gamma(x)$, and

$$
D_{2}\left[\mu x[s] \rrbracket(\gamma)=\lim _{i} p_{i}, \text { where } p_{0}=\{1\}\right. \text { and }
$$$$
P_{i+1}=D_{2} \llbracket s \rrbracket\left(\gamma<p_{i} / x>\right)
$$

4.4. Relationship between $\mathrm{O}_{2}$ and $D_{2}$.

we shall show that

$$
\text { (*) } o_{2}=\alpha_{2} \circ D_{2} \text {, }
$$

for suitable $\alpha_{2}$. In fact, $\alpha_{2}$ is defined in two steps:

1. First we define $s y n_{2}: P \rightarrow P$ for $p \in P_{\omega}$

$$
\begin{aligned}
\operatorname{syn}_{2}(p)= & \{a \mid a \in p \text { and } a \notin c\} u \\
& \left\{\left[a, s y n_{2}(q)\right] \mid[a, q] \in p \text { and } a \notin c\right\}
\end{aligned}
$$

For $p \in P \backslash P_{\omega^{\prime}}$ we have $p=\lim _{n} p_{n}$, with $P_{n} \in P_{n^{\prime}}$, and we put $\operatorname{syn}_{2}(p)=1 \mathrm{~m}_{\mathrm{n}}\left(\operatorname{syn}_{2}\left(\mathrm{p}_{\mathrm{n}}\right)\right)$.

Example. Let $\mathrm{p}=\mathcal{D}_{2} \mathbb{I}(\mathrm{a}+\mathrm{c}) \|(\mathrm{b}+\overline{\mathrm{c}}) \rrbracket$. Then $\operatorname{syn}_{2}(p)=\{[a,\{b\}],[b,\{a\}], \tau\}$.

2. Next, we define traces: $P \rightarrow P\left(A_{\delta}^{t r}\right)$ by (finite case only displayed) :

$$
\text { traces } \begin{array}{rlrl}
(p) & =U\{\text { traces }(x): x \in p\} & \text { if } p \neq \emptyset \\
& =\{\delta\} & & \text { if } p=\emptyset
\end{array}
$$

where $\operatorname{traces}(a)=\{a\}, \operatorname{traces}([a, q])=\operatorname{artraces}(q)$. We now put

$$
\alpha_{2}=\text { df. } \text { traces o syn }_{2} \text {, }
$$

but we cannot (yet) prove (*), because, similarly to $\alpha_{1}, \alpha_{2}$ does not behave homomorphically.

Therefore, we try an intermediate semantics $I_{2}$. This cannot be based on a simple IT model as the following argument shows:

Let us try for $I_{2}$, similarly to $I_{1}$, the addition of the axiom $\langle c, w\rangle \rightarrow$ w.c to $T_{2}$. Now consider the programs $s_{1} \equiv a_{i}\left(c_{1}+c_{2}\right), s_{2} \equiv\left(a ; c_{1}\right)+\left(a ; c_{2}\right)$, $\mathrm{s} \equiv \bar{c}_{1}$. Then $O_{2}\left[\mathrm{Is}_{1} \| \mathrm{s} \Pi=\{\mathrm{a} T\} \neq\{\mathrm{a} \tau, \mathrm{a} \delta\} \rightleftharpoons\right.$ $0_{2} \llbracket s_{2} \| s \rrbracket$. However, $I_{2} \llbracket s_{1}\left\|s \rrbracket=I_{2} \llbracket s_{2}\right\| s \rrbracket$. Thus whatever $\alpha$ we apply to $I_{2}[1$.$] , the results for$ $s_{1}\left\|s, s_{2}\right\| s$ will turn out the same.

our solution to this problem is to introduce an. intermediate semantics $I_{2}$ which, besides recording all traces in $A_{\delta}^{t r}$, also records a very weak information about the local branching structure of the process. This information is called a ready set or deadlock possibility: it is a subset $\mathrm{x}$ of c. Informally, $x$ indicates the set of communications $c$ which are ready to synchronize with any other matching communication $\bar{c}$ from another parallel compound (for the notion of ready set cf. $[8,11,18,19,21])$. Formally, take $\Delta=P(C)$. For $\mathrm{x} \in \Delta$, let $\overline{\mathrm{X}}=\{\overrightarrow{\mathrm{c}} \mid \mathrm{c} \in \mathrm{x}\}$. The ready domain $R$ is now $R=P\left(A^{t r} \cup A^{t r} \cdot \Delta\right)$. The transition system $T_{2}^{*}$ consists of all axioms and rules of $\mathrm{T}_{2}$ together with (for $w \in A^{*}$ ).

$$
\begin{aligned}
& \text { (i) }\langle\mathrm{c}, w\rangle \rightarrow w \cdot c \\
& \text { (ii) }\langle\mathrm{c}, w\rangle+w \cdot\{c\} \\
& \text { (iii) }\langle\text { fail, w } \rightarrow w \cdot \emptyset \\
& \text { (iv) } \frac{\left\langle\mathrm{s}_{1}, w\right\rangle+\mathrm{w}, \mathrm{X},\left\langle\mathrm{s}_{2}, \mathrm{w}\right\rangle \rightarrow \mathrm{w} \cdot \mathrm{Y}}{\left\langle\mathrm{s}_{1}+\mathrm{s}_{2}, \mathrm{w}\right\rangle+\mathrm{w} \cdot \mathrm{XUY}}
\end{aligned}
$$




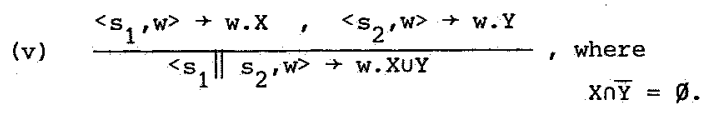

Axioms (ii), (iii) introduce deadlock

possibilities/ready sets. Rule (iv) says that

$s_{1}+s_{2}$ has a (one-step) deadlock possibility only

if $s_{1}$ and $s_{2}$ have, and rule $(v)$ says that $s_{1} \| s_{2}$ has a (one-step) deadlock possibility if both $s_{1}$ and $s_{2}$ have, and no synchronization is possible. We omit the natural definition of $\mathrm{I}_{2}$ from $\mathrm{T}_{2}^{*}$.

Examples ( $I_{2}$ semantics)

(i) $I_{2}[\mathrm{a} ;(\mathrm{b}+\mathrm{c}) \Pi=\{\mathrm{ab}, \mathrm{ac}\}$.

Proof. We explore all transition sequences

in $\mathrm{T}_{2}^{*}$ starting in $\langle\mathrm{a} ;(\mathrm{b}+\mathrm{c}), \lambda\rangle$ :
(1) $\langle a, \lambda\rangle \rightarrow a$
(elem.action)
(2) $\langle\mathrm{a} ;(\mathrm{b}+\mathrm{c}), \lambda\rangle \rightarrow\langle\mathrm{b}+\mathrm{c}, \mathrm{a}\rangle$
(seq. comp.: (1))
(3) $\langle$ b. a $\rangle+a b$
(elem.action)
(4) $\langle c, a\rangle \rightarrow a c$
a. $\{c\}$
(comm.)

(5) $\langle\mathrm{b}+\mathrm{c} \cdot \mathrm{a}\rangle \underset{\mathrm{ac}}{\rightarrow} \mathrm{ab}$

(glob. nondet.

(3), (4))

No more transitions are

deducible for $\langle b+c, a\rangle$.

(6) Thus *

$\begin{aligned}\langle a ;(b+c), \lambda\rangle \rightarrow\langle b+c, a\rangle & \rightarrow a b \\ & \geqslant a c\end{aligned}$

are all transition sequences

starting in $\langle a ;(b+c), \lambda\rangle$.

This proves the claim

(ii) $I_{2}[a ; b+a ; c]=\{a b, a c, a,\{c\}\}$.

Proof. Here we only exhibit all possible

transition sequences in $\mathrm{T}_{2}^{*}$ starting in

$\langle a ;(b+c), \lambda>$ :

$\begin{aligned}\langle a ; b+a ; c, \lambda\rangle & \rightarrow\langle b, a\rangle \rightarrow a b \\ \forall<c, a\rangle & \rightarrow a c \\ & \forall a .\{c\}\end{aligned}$
LEMMA 4.1. For all $\mathrm{s}^{\mathrm{s}^{\prime}} \epsilon(\mathrm{A} \backslash \mathrm{C})^{*}$ the following holds:

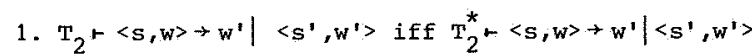

2. $\langle s, w\rangle$ is a deadiocking configuration for $\mathrm{T}_{2}$ iff there exists some $\mathrm{X} \subseteq \mathrm{C}$ with $\mathrm{T}_{2}^{*}{ }^{*}\langle s, w>+\mathrm{w} \cdot \mathrm{x}$. Let now $w$ range over $A^{t r}=A^{*} \cup A^{\omega} \cup A^{*} .\{1\}$ and let w range over $R=P\left(A^{\text {tr }} \cup A^{t r} \cdot \Delta\right)$. We define the abstraction operator $\operatorname{syn}_{2}^{*}: R+P\left(A_{\delta}^{t x}\right)$ by $\operatorname{syn}_{2}^{*}(w)=\{w \mid w \in w$ does not contain any $c \in c\} u$

$\{w \delta \mid \exists x \in \Delta: w . X \in W\}$

We have

THEOREM 4.2. $O_{2}=\operatorname{syn}_{2}^{*} \circ I_{2}$. Next, we wish to relate $I_{2}$ with the full BT semantics $D_{2}$. To this end, we introduce the abstraction operator readies: $P+R$ by defining readies ( $p$ ) as follows (finite case only). Let $p=\left\{a_{1}, \ldots, a_{m},\left[b_{1}, q_{1}\right], \ldots,\left[b_{n}, q_{n}\right]\right\}$, with $a_{i}, b_{j} \in A$. We put readies $(\mathrm{p})=U\{$ readies $(\mathrm{x}): \mathrm{x} \in \mathrm{p}\} U$

$$
\left\{\lambda . x \mid x=\left\{a_{1}, \ldots, a_{m}, b_{1}, \ldots, b_{n}\right\} \subseteq c\right\}
$$

where readies $\left(a_{i}\right)=\left\{a_{i}\right\}$, readies $\left(\left[b_{j}, a_{j}\right]\right)=$ $\mathrm{b}_{j}$.readies $\left(\mathrm{q}_{\mathrm{j}}\right)$.

THEOREM 4.3. $I_{2}=$ readies $\circ D_{2}$.

Proof. (i) readies behaves homomorphically on ,$;+, \|$. (ii) $I_{2}(\mu x[s])$ can be obtained by applying readies to the fixed point definition of $\mu x[s]$ under $D_{2}$.

LEMMA 4.4. traces $\operatorname{syn}_{2}=\operatorname{syn}_{2}^{*}$. readies summarizing, we have our final

THEOREM 4.5. $\mathrm{O}_{2}=$ traces $\circ \operatorname{syn}_{2} \circ \mathrm{D}_{2}$.

For the further results the following lemma is important: 


\section{REFERENCES}

[1] K.R. Apt (1981), Recursive Assertions and Parallel Programs, Acta Inf. 15, pp.219232.

[2] K. R. Apt (1983), Formal Justification of a Proof System for Communicating Sequential Processes, J. Assoc. Comput. Mach.,30(1), pp. 197-216.

[3] J.W. de Bakker, J.A. Bergstra, J.W. Klop \& J.-J.Ch. Meyer (1983), Linear Time and Branching Time Semantics for Recursion with Merge, Theoretical Computer Science 34 (1984), pp.135-156.

[4] J.W. de Bakker \& J.I. Zucker (1982), Denotational Semantics of Concurrency, in Proceedings 14th Assoc.Comput.Mach. Symp. on Computing, pp.153-158.

[5] J.W. de Bakker \& J.I. Zucker (1982), Processes and the Denotational Semantics of Concurrency, Inform. and Control 54 (1/2), pp. 70-120.

[6] J.w. de Bakker \& J.I. Zucker (1983), Compactness in Semantics for Merge and Fair Merge, in: E. Clarke \& D. Kozen (eds.), Proceedings Workshop Logics of Programs, Pittsburgh, Springer LNCS, 164, pp. 18-33.

[7] J.A. Bergstra \& J.W. Klop (1984), Process Algebra for Synchronous Communication, Information and Control 60 (1984),pp.109-137.

[8] S.D. Brookes, C.A.R. Hoare \& A.W. Roscoe (1984), A Theory of Communicating Sequential Processes, J.Assoc.Comput. Mach. 31 (3), pp.560-599.

[9] M. Broy (1983), Fixed Point Theory for Communication and concurrency, in: D. Bjorner (ed.), Proceedings IFIP Working Conference on Formal Description of Programming Concepts II, North-Holland Amsterdam, pp.125-148.

[10] J. Dugundji (1966), Topology, Allen and Bacon, Rockleigh, N.J.

[11] N. Francez, D.J. Lehmann \& A. Pnueli (1984), A Linear-History Semantics for Languages for Distributed Programing, Theor.Comp. Sc. $32(1 / 2)$, pp. 25-46.

[12] W.G. Golson \& w.C. Rounds (1983), Connections between Two Theories of concurrency:
Metric Spaces and Synchronization Trees, Inform. and Control 57(2/3), pp.102-124.

[13] M. Hennessy \& G.D. Plotkin (1979), Full Abstraction for a Simple parallel Programming Language, in: J. Bečvar̆ (ed.), Proceedings 8 th MFCS, tNCS 74, Springer, Berlin/New York, pp.108-120. ,

[14] R. Keller (1976), Formal verification of Parallel programs, Comm. Assoc. Comput. Mach. 19, pp.371-384.

[15] J.-J.Ch. Meyer (1984), Fixed Points and the Axbitrary and Fair Merge of a Fairly Simple Class of Processes, Techn. Reports IR-89/IR-92. Free University, Amsterdam.

[16] R. Milner (1980), A Calculus for Communicating Systems, LNCS 92, Springer, Berlin/New York

[17] M. Nivat (1979), Infinite Words, Infinite Trees, Infinite Computations, in Proceedings Found. of Comp. Sc. III.2, Mathematical Centre Tracts 109, Amsterdam, pp.3-52.

[18] E. -R. Olderog \& C.A.R. Hoare (1983), Specification-oriented semantics for Communicating Processes in: J. Diaz (ed.), Proceedings 10th Int. Coll. on Autom., Langu. and Programming, pp.561-572.

[19] E.-R. Olderog \& C.A.R. Hoare (1984), Specification-oriented Semantics for Communicating Processes, Techn. Monograph PRG-37, Oxford Univ. Progr. Research Group.

[20] G.D. Plotkin (1983), An Operational Semantics for CSP in: D. Bjorner (ed.), Formal Description of Programming Concepts II, North-Holland, Amsterdam, pp.199-223.

[21] W.C. Rounds \& S.D. Brookes (1981), Possible Futures, Acceptances, Refusals, and Communicating Processes, in Proceedings 22nd Symp. Found. of Comp. Sc., IEEE, pp.140-149. 